\title{
Performed Composition Relationship
}

National Cancer Institute

\section{Source}

National Cancer Institute. Performed Composition Relationship. NCI Thesaurus. Code C153108.

A relationship between a composite activity and a component activity that comprises it, i.e. parent and child activities where all activities have occurred in the context of a particular study or experiment. 